\title{
Karachay-Balkar Language
}

National Cancer Institute

\section{Source}

National Cancer Institute. Karachay-Balkar Language. NCI Thesaurus. Code C153987.

A T urkic language spoken by the Karachay and Balkar people in the Kabardino-Balkaria and Karachay-Cherkessia republics of Russia. 